\title{
Transient Solutions for Stratified Fluid Flows*
}

\author{
David W. Fox**
}

(May 14, 1975)

\begin{abstract}
The solution to an initial value problem for the flow of a buoyant inviscid incompressible fluid in three-space is given in terms of a fundamental solution. The initial values and the distribution of source strength can be quite general subject only to mild restrictions on smoothness. The fundamental solution and its associated velocity and displacement fields are given explicitly and in some detail.
\end{abstract}

Key words: Fluid dynamics; fundamental solution, initial value problem; partial differential equations; stratified flow, internal waves, buoyant flows.

\section{Introduction}

This article presents a solution to an initial value problem in the theory of stratified fluids. Thus it is a contribution to the theory of buoyant flow and internal gravity waves and also to the closely related topic of flows in rotating fluids. ${ }^{1} \mathrm{~A}$ system of two linear second order equations in three space dimensions and time govern the problem. The equations describe the flow of an inviscid incompressible fluid in which an arbitrary distribution of source strength is prescribed. The approximations that the density and the buoyancy frequency are constant are used. A fundamental solution of the system is used to solve the initial value problem in terms of the source distribution and the initial conditions. The fundamental solution is given explicitly; and a number of its properties are noted, including its velocity and displacement fields.

Section 1 gives a summary sketch of the system of equations governing the flow. They result from a first order perturbation from static equilibrium of the conservation equations for momentum, density, and mass. Needless to say, these equations agree in all respects with those given in standard works such as [8]. ${ }^{2}$ Two technical differences are worth noting. First, the unknown functions in the system with which we work differ somewhat from those most often seen; and second, we prefer to deal with a pair of second order differential equations rather than combining them into a single fourth order equation. This section concludes with the statement of an appropriate set of initial conditions for the problem.

We start section 2 by writing down the full initial-value problem in the exact form we need. Then in section 2.1 we give the fundamental solution and an interpretation of it as an impulsive injection of fluid; the corresponding velocity field is also presented. A verification of the solution is sketched. In section 2.2 the solution of the problem with a prescribed source distribution but zero initial conditions is given. Then the initial conditions are accounted for, and everything is put together. Some of the properties of the solutions are verified in this section, but other technical details will appear elsewhere [3].

Properties of the solutions are examined in section 3. The structure of the fundamental solution shows a natural decomposition of the flow into two parts, one the flow of nonbuoyant ideal fluid of constant density and the other a modification that gives the influence of buoyancy. The first part

\footnotetext{
* An invited paper. This work supported by the Department of the Navy, Naval Sea Systems Command under Contract No. N00017-72-C-4401.

** Present address: Applied Physics Laboratory, The Johns Hopkins University, Laurel, Maryland 20810.

1 These topics are in rapid development. For recent work the reader should look at the last several years of the Journal of Fluid Mechanics, Geophysical Fluid Dynamics, Physics of Fluids, and the references given there.

${ }^{2}$ Figures in brackets indicate the literature references at the end of this paper.
} 
responds immediately to the injection, the second is slow and long lasting. We give especially simple expressions for the fundamental solution directly above and below the singular point and in the horizontal plane with it.

The solutions of the initial-value problem are then examined in light of the properties of the fundamental solution. We note that, in addition to an oscillating source-like effect, the initial conditions produce a term that rings without changing spatial distribution. Under certain conditions this can be the total effect of the initial conditions. In other circumstances, when the initial conditions are confined to a bounded region, we show that the influence of the initial conditions dies off at large distances faster than that of a source distribution with nonzero total strength.

The last section discusses very quickly the displacements that result from the fundamental solution. These are purely radial and are found from an exact integration of the velocities.

A summary of portions of this work has been presented in [2].

\section{The Equations of Motion}

We start from a usual system of equations for an inviscid incompressible fluid in a constant gravity field. These state the conservation of momentum, density (incompressibility), and mass:

$$
\begin{array}{r}
\rho D \mathbf{u} / D t+\operatorname{grad} p+g \rho \mathbf{k}=0, \\
D \rho / D t=0, \\
\rho \operatorname{div} \mathbf{u}=\mu,
\end{array}
$$

where $\mathbf{k}$ is the vertical unit vector (in the $x_{3}$-direction), and $\mu$ is a prescribed distribution of source strength. The rest of the notation is standard. These equations are to be linearized about a state of stable static equilibrium described by $\mathbf{u}=\mathbf{u}_{0}=0, \rho=\rho_{0}\left(x_{3}\right)$, and $p=p_{0}=-g \int_{0}^{x_{3}} \rho_{0}(z) d z$. For stability $\rho_{0}$ is required to be positive and monotone decreasing with $x_{3}$. The perturbed flow is taken in the form $\mathbf{u}=\mathbf{u}_{0}+\widetilde{\mathbf{u}}, \rho=\rho_{0}+\widetilde{\rho}$, and $p=p_{0}+\widetilde{p}$. These are put in (1.1), (1.2), and (1.3), and quantities of order higher than the first are discarded to obtain the linear equations

$$
\begin{aligned}
\rho_{0} \partial \widetilde{\mathbf{u}} / \partial t+\operatorname{grad} \widetilde{p}+g \widetilde{\rho} \mathbf{k} & =0, \\
\partial \widetilde{\rho} / \partial t+\widetilde{u}_{3} d \rho_{0} / d x_{3} & =0, \\
\rho_{0} \operatorname{div} \widetilde{\mathbf{u}} & =\mu .
\end{aligned}
$$

Now we drop the ${ }^{\sim}$ 's, and henceforth all the quantities will be the perturbations except for $\rho_{0}$, which designates the prescribed equilibrium density. From the horizontal components of (1.4) it follows that the vertical component of vorticity does not change with time so that for a variety of flows, including all motions that start from rest, the horizontal velocity components derive from a potential. We assume this to be the case. For convenience we write this in the form

$$
u_{\alpha}=\frac{1}{\rho_{0}} \frac{\partial \varphi}{\partial x_{\alpha}}, \quad \alpha=1,2
$$

Also from (1.4) and (1.7) it follows that $p$ can be expressed by

$$
-p=\partial \varphi / \partial t
$$

and that the equation of motion in the vertical direction takes the form 


$$
\frac{\partial}{\partial t}\left(\rho_{0} u_{3}-\frac{\partial \varphi}{\partial x_{3}}\right)+g \rho=0
$$

The equations (1.9) and (1.5) imply

$$
\frac{\partial^{2}}{\partial t^{2}}\left(u_{3}-\frac{1}{\rho_{0}} \frac{\partial \varphi}{\partial x_{3}}\right)-\frac{g}{\rho_{0}} \frac{d \rho_{0}}{d x_{3}} u_{3}=0
$$

and (1.6) takes the form

$$
\frac{\partial^{2} \varphi}{\partial x_{1}^{2}}+\frac{\partial^{2} \varphi}{\partial x_{2}^{2}}+\rho_{0} \frac{\partial u_{3}}{\partial x_{3}}=\mu
$$

Now we introduce the notation $n$ and $w^{*}$. The buoyancy frequency $n$ is defined by $n^{2} \equiv-\left(g / \rho_{0}\right) d \rho_{0} /$ $d x_{3}$, and $w^{*}$ is given by

$$
w^{*} \equiv u_{3}-\frac{1}{\rho_{0}} \frac{\partial \varphi}{\partial x_{3}}
$$

We write the preceding pair of equations in the more convenient forms

$$
\left(\frac{\partial^{2}}{\partial t^{2}}+n^{2}\right) w^{*}+\frac{n^{2}}{\rho_{0}} \frac{\partial \varphi}{\partial x_{3}}=0
$$

and

$$
\frac{\partial^{2} \varphi}{\partial x_{1}^{2}}+\frac{\partial^{2} \varphi}{\partial x_{2}^{2}}+\rho_{0} \frac{\partial}{\partial x_{3}}\left(\frac{1}{\rho_{0}} \frac{\partial \varphi}{\partial x_{3}}\right)+\rho_{0} \frac{\partial w^{*}}{\partial x_{3}}=\mu .
$$

Note that in general $n^{2}$ and $\rho_{0}$ depend on $x_{3}$.

The coupled system (1.11) and (1.12) governs the quantities $\varphi$ and $w^{*}$, from which in turn the eqs (1.7), (1.8), (1.9) and the definition (1.10) determine the velocities, density, and pressure. However the system must be completed by appropriate boundary conditions for $\varphi$ and $w^{*}$ on the prescribed boundaries, a surface condition on free surfaces, and by initial conditions.

Here we shall be concerned with the situation in which all of the boundaries are far away from the region of interest and their influence can be neglected. In this case only initial conditions remain to be considered. These fall on $w^{*}$ alone since in (1.11) and (1.12) $w^{*}$ is the only quantity to be differentiated with respect to $t$. They can be prescribed by

$$
w^{*}\left(x, 0^{+}\right)=f(x) \quad \text { and } \quad \frac{\partial w^{*}}{\partial t}\left(x, 0^{+}\right)=g(x)
$$

where $f$ and $g$ are given functions. The second of (1.13) is equivalent to a statement of the initial perturbation density as can be seen from (1.9) and (1.10).

It may seem strange at first to give initial conditions on just $w^{*}$ and $\partial w^{*} / \partial t$, but this is precisely what the system (1.11) and (1.12) requires. This is to be compared with a similar circumstance in the linear theory of surface gravity waves on an ideal fluid in which the initial values of the potential and its time derivative on the free surface are all that is required. ${ }^{3}$ In each case the reason is much 
the same: an equation of motion, in our case (1.12), determines the remainder of the solution at $t=0^{+}$from what is given.

\section{An Initial Value Problem}

Here we present a solution to a general initial-value problem for a fluid in which the density and the buoyancy frequency are treated as constant, i.e., the flow is described by (1.11) and (1.12) in which $\rho_{0}$ and $n$ are taken to be constants. The region of the solution is all of three-space.

We shift the time scale to $\bar{t}=n t$, set $\bar{g}=g / n$, note that $\bar{n}=n / n=1$, and then drop the ${ }^{-{ }^{\prime}}$ s for simplicity; the problem is thereby put in the form

$$
\left.\begin{array}{r}
\left(\frac{\partial^{2}}{\partial t^{2}}+1\right) w^{*}+\frac{1}{\rho_{0}} \frac{\partial \varphi}{\partial x_{3}}=0 \\
\nabla^{2} \varphi+\rho_{0} \frac{\partial w^{*}}{\partial x_{3}}=\mu,
\end{array}\right\} x, t \epsilon R^{3} \times(0, \infty)
$$

where $\nabla^{2}$ is the three-dimensional Laplacian. The initial conditions become

$$
w^{*}\left(x, 0^{+}\right)=f(x), \quad \frac{\partial w^{*}}{\partial t}\left(x, 0^{+}\right)=g(x) .
$$

Our approach to the solution of the problem stated by $(2.1)$ and (2.2) is the determination and systematic use of a fundamental solution of (2.1).

The importance of a fundamental solution derives from the fact that for the equations and region for which it is valid, it reduces the solution of inhomogeneous initial-value problems to integration. Further, by a variety of more-or-less standard devices it often can be modified to apply to a number of other regions. Equally important, an analysis of the behavior of a fundamental solution can yield considerable information on the properties of whole classes of solutions, any of which might be difficult to obtain explicitly. In addition, it is a usual starting point for a formulation of initial-boundary-value problems in terms of integral equations.

\subsection{A Fundamental Solution}

A fundamental solution for the problem stated by (2.1) and (2.2) is a singular solution $\hat{\varphi}, \hat{w}^{*}$ of (2.1) that gives the effect at $x, t$ of a disturbance at $y, \tau$. Here the disturbance can be interpreted as an impulsive injection at time $\tau$ of a unit mass of fluid of density $\rho_{0}$ at the point $y$. The body of fluid is supposed to be at rest prior to the injection. Since the fundamental solution will depend only on $x-y$ and $t-\tau$, we need to consider only $y=0$ and $\tau=0$ for the moment.

The fluid is at rest prior to $t=0$, and consequently both $\hat{\varphi}$ and $\hat{w}^{*}$ will be identically zero for $t<0$. For $t \geqslant 0$ our fundamental solution is given by

$$
\begin{aligned}
\hat{\varphi}(x, t) & =\frac{-1}{4 \pi|x|}\left\{\delta(t)+\frac{\partial}{\partial t} J_{0}(t \cos \theta)+\left[J_{0}(t \cos \theta)\right]^{*}\left[\frac{J_{1}(t)}{t}\right]\right\} \\
\hat{w}^{*}(x, t) & =\frac{-1}{4 \pi \rho_{0}|x|^{2}}\left\{\frac{\partial}{\partial t} t J_{1}(t \cos \theta)+\left[t J_{1}(t \cos \theta)\right]^{*}\left[\frac{J_{1}(t)}{t}\right]\right\}
\end{aligned}
$$

where $|x|=\left(x_{1}^{2}+x_{2}^{2}+x_{3}^{2}\right)^{1 / 2}, \delta(t)$ is the Dirac $\delta$-measure concentrated at zero, and $\cos \theta=x_{3} /|x|$. 
The symbol * indicates convolution, e.g., $l(t) * m(t) \equiv \int_{0}^{t} l(\tau) m(t-\tau) d \tau$; and $J_{0}$ and $J_{1}$ are Bessel functions of the first kind. The fundamental velocities $\hat{\mathbf{u}}$ are those corresponding by (1.7) and (1.10) to the fundamental solution (2.3). These formulas yield by a direct calculation.

$$
\hat{\mathbf{u}}=\frac{x}{4 \pi \rho_{0}|x|^{3}}\left\{\delta(t)+\frac{\partial^{2}}{\partial t^{2}} t J_{0}(t \cos \theta)+\left[\frac{\partial}{\partial t} t J_{0}(t \cos \theta)\right] *\left[\frac{J_{1}(t)}{t}\right]\right\}
$$

In the computation it is economical to differentiate the quantity in braces in $\hat{\varphi}$ with respect to $\cos \theta$ and then $\cos \theta$ with respect to each of the space variables.

We give elsewhere [3] a detailed and mathematically complete demonstration of the validity of the fundamental solution and the representation formulas that make use of it. Here we give only a very brief sketch.

Note first that $\hat{\varphi}, \hat{w}^{*}$, and $\hat{\mathbf{u}}$ given by (2.3) and (2.4) have the correct coordinate singularities for a fundamental solution [1]. It is quite easy to show that $\hat{\varphi}$ and $\hat{w}^{*}$ do satisfy (2.1) with $\mu$ equal to zero for any nonzero $x$. In fact, the first of (2.1) is demonstrated by showing that $\partial^{2} w^{*} / \partial t^{2}$ is just the negative of the third component of $\hat{\mathbf{u}}$ given by (2.4), and consequently equal by (1.10) to $-\left(\hat{w}^{*}+\frac{1}{\rho_{0}} \frac{\partial \hat{\varphi}}{\partial x_{3}}\right) \cdot$ Note that the $\delta(t)$ appears in the second differentiation of $\hat{w}^{*}$ due to the jump in $\partial \hat{w}^{*} / \partial t$ at $t=0$. The verification of the second equation of (2.1) is equivalent to showing that $\rho_{0} \operatorname{div}$ $\hat{\mathbf{u}}=0$ for $x \neq 0$. This is accomplished by a routine calculation on (2.4) using the same device as in the computation of $\hat{\mathbf{u}}$. The vanishing of $\rho_{0} \operatorname{div} \hat{\mathbf{u}}$ for $x \neq 0$ implies that the mass outflow $\hat{Q}(t)$ from the origin can be calculated from $\hat{Q}(t)=\rho_{0} \int_{S} \hat{\mathbf{u}} \cdot \mathbf{n} d s$ for any fixed closed surface $S$ that contains the origin. Taking $S$ to be the sphere $|x|=1$ we find $\hat{Q}(t)=\delta(t)$. The computation is aided by using polar coordinates, observing that $\int_{0}^{\pi} J_{0}(t \cos \theta) \sin \theta d \theta=\frac{2}{t} \int_{0}^{t} J_{0}(\tau) d \tau$, and using the relation ${ }^{4}$ $\left[J_{0}(t)\right]^{*}\left[J_{1}(t) / t\right]=J_{1}(t)$. Notice that only the leading term in (2.4) gives a nonzero contribution to $\hat{Q}(t)$.

\subsection{Representation of Solutions}

The fundamental solution (2.3) provides a representation for solutions of the initial value problem (2.1) and (2.2) with only mild restrictions on $\mu, f$, and $g$.

To begin we consider the case with $f$ and $g$ equal to zero. Then $\varphi$ and $w^{*}$ are given by the integral formulas.

$$
\left.\begin{array}{c}
\varphi(x, t)=\int_{0}^{t} \int_{R^{3}} \hat{\varphi}(x-y, t-\tau) \mu(y, \tau) d y d \tau, \\
w^{*}(x, t)=\int_{0}^{t} \int_{R^{3}} \hat{w}^{*}(x-y, t-\tau) \mu(y, \tau) d y d \tau .
\end{array}\right\}
$$

The parallel formula for the velocity is

$$
\mathbf{u}(x, t)=\int_{0}^{t} \int_{R^{3}} \hat{\mathbf{u}}(x-y, t-\tau) \mu(y, \tau) d y d \tau .
$$

The verification that the velocity derived from (2.5) by the formulas (1.7) and (1.10) is that given by (2.6) and that the equations (2.1) are satisfied by (2.5) is given in [3]. The arguments there

${ }^{4}$ See [9] p. 380. 
are principally of the type used in [5] to demonstrate the differentiability properties of generalized domain potentials for elliptic partial differential equations. A suitable set of conditions to impose on $\mu$ are that it be piecewise continuous in $t$ for $t \geqslant 0$ for all $x$ and satisfy a uniform Lipschitz condition in $x$ for all $t \geqslant 0$. These are sufficient for the proofs in [3], but certainly not necessary.

On the other hand it is comparatively easy to see directly that $w^{*}$ given by (2.5) satisfies $w^{*}\left(x, 0^{+}\right)=\frac{\partial w^{*}}{\partial t}\left(x, 0^{+}\right)=0$. In fact, the expression for $\hat{w}^{*}$ in (2.3) shows that for small positive $t$

$$
\hat{w}^{*}=-\frac{1}{4 \pi \rho_{0}} \frac{x_{3}}{|x|^{3}} t\left[1+t^{2} B(\cos \theta, t)\right]
$$

where $B$ is uniformly bounded in $t$ and $\cos \theta$. This implies that $w^{*}(x, t)$ given by $(2.5)$ is $0\left(t^{2}\right)$ provided only that $\mu(x, t)$ is not too rough, e.g., continuous and uniformly bounded, for small nonzero $t$. From this the required initial conditions follow immediately.

Now suppose $\mu \equiv 0$ but that the initial functions $f$ and $g$ for $w^{*}$ and $\partial w^{*} / \partial t$ are nonvanishing. We require that $\partial f / \partial x_{3}$ and $\partial g / \partial x_{3}$ exist and satisfy the same sort of smoothness conditions given for $\mu$ earlier. Let $h(x, t)$ be defined by

$$
h(x, t)=\rho_{0}[f(x) \cos t+g(x) \sin t] .
$$

Then a solution to (2.1) with (2.2) is given by

$$
\begin{aligned}
& \varphi(x, t)=-\int_{0}^{t} \int_{R^{3}} \hat{\varphi}(x-y, t-\tau) \quad \frac{\partial h}{\partial y_{3}}(y, \tau) d y d \tau, \\
& \left.w^{*}(x, t)=-\int_{0}^{t} \int_{R^{3}} \hat{w}^{*}(x-y, t-\tau) \frac{\partial h}{\partial y_{3}}(y, \tau) d y d \tau+\frac{h(x, t)}{\rho_{0}},\right\}
\end{aligned}
$$

and the corresponding velocity is

$$
\mathbf{u}(x, t)=-\int_{0}^{t} \int_{R^{3}} \hat{\mathbf{u}}(x-y, t-\tau) \frac{\partial h}{\partial y_{3}}(y, \tau) d y d \tau+\frac{h(x, t) \mathbf{k}}{\rho_{0}} .
$$

Since the integral expression in $w^{*}$ given by (2.7) and its derivative with respect to $t$ vanish at $t=0$, the function $w^{*}$ has the same initial conditions as $h / \rho_{0}$ as required. Further, since $h$ satisfies $\partial^{2} h / \partial t^{2}+h=0$, the functions $\phi$ and $w^{*}$ satisfy the first of $(2.1)$. The second is also satisfied with $\mu \equiv 0$ since the contribution from the integrals in the solution is exactly cancelled by the contribution from the nonintegral term in $w^{*}$.

By linearity the sum of the solution given by (2.5) and that of (2.7) satisfy the full inhomogeneous initial-value problem (2.1), (2.2); the corresponding velocities are the sum of (2.6) and (2.8). Thus a full solution of $(2.1),(2.2)$ is given by

$$
\left.\begin{array}{rl}
\varphi(x, t) & =\int_{0}^{t} \int_{R^{3}} \hat{\varphi}(x-y, t-\tau)\left[\mu(y, \tau)-\frac{\partial h}{\partial y_{3}}(y, \tau)\right] d y d \tau, \\
w^{*}(x, t) & =\int_{0}^{t} \int_{R^{3}} \hat{w}^{*}(x-y, t-\tau)\left[\mu(y, \tau)-\frac{\partial h}{\partial y_{3}}(y, \tau)\right] d y d \tau+\frac{h(x, t)}{\rho_{0}},
\end{array}\right\}
$$

and

$$
\mathbf{u}(x, t)=\int_{0}^{t} \int_{R^{3}} \hat{\mathbf{u}}(x-y, t-\tau)\left[\mu(y, \tau)-\frac{\partial h}{\partial y_{3}}(y, \tau)\right] d y d \tau+\frac{h(x, t) \mathbf{k}}{\rho_{0}} .
$$




\section{Properties of the Solutions}

Here we examine some of the properties of the solutions of the initial-value problem (2.1), (2.2). We begin by pointing out some of the more evident properties of the fundamental solution (2.3) and the fundamental velocities (2.4).

By inspection of the expressions for $\hat{\varphi}$ and $\hat{\mathbf{u}}$ it is clear that each of them breaks naturally into two parts; the first is exactly that of the fundamental solution in $R^{3}$ for an ideal fluid of constant density $\rho_{0}$, and the remaining part the required modifications which account for the effects of buoyancy. Thus the potential and velocities can be conveniently written as $\hat{\varphi}=\hat{\varphi}^{\circ}+\hat{\varphi}^{*}$ and $\hat{\mathbf{u}}=$ $\hat{\mathbf{u}}^{\circ}+\hat{\mathbf{u}}^{*}$, where

$$
\begin{gathered}
\hat{\varphi}^{*}(x, t)=-\frac{1}{4 \pi|x|}\left\{\frac{\partial}{\partial t} J_{0}(t \cos \theta)+\left[J_{0}(t \cos \theta)\right]^{*}\left[\frac{J_{1}(t)}{t}\right]\right\} \\
\hat{\mathbf{u}}^{*}(x, t)=\frac{x}{4 \pi \rho_{0}|x|^{3}}\left\{\frac{\partial^{2}}{\partial t^{2}} t J_{0}(t \cos \theta)+\left[\frac{\partial}{\partial t} t J_{0}(t \cos \theta)\right] *\left[\frac{J_{1}(t)}{t}\right]\right\}
\end{gathered}
$$

Examination of $\hat{\varphi}^{*}$ and $\hat{\mathbf{u}}^{*}$ shows that each of them is $0(t)$ at $t=0$, and consequently the influence of buoyancy takes some time to have an important effect; however that effect is long lasting, as we shall see more fully later. Since the quantities in braces in (3.1) are bounded functions of $\cos$ $\theta$ for each $t$, it is clear that the velocity field decays in space like $1 /|x|^{2}$, i.e., just as in the ideal fluid case.

As we have noted earlier, the fundamental velocity field $\hat{\mathbf{u}}$ has vanishing divergence except at $x=0$ and has a net mass outflow $\delta(t)$ from the origin, but the same is true of the ideal fluid part $\hat{\mathbf{u}}^{\circ}$. This means that the buoyancy induced velocity $\hat{\mathbf{u}}^{*}$ is also divergence free and, while singular at the origin, has zero net mass outflow.

The symmetry properties of the fundamental velocity $\hat{\mathbf{u}}$ are those to be expected: axial symmetry about the $x_{3}$ axis and odd symmetry about the origin. It is perhaps more surprising that $\hat{\mathbf{u}}$ is purely radial. Since it is, and is also proportional to $1 / r^{2}$, the corresponding fundamental displacements are also purely radial and can be calculated easily.

We now turn our attention to those places where the fundamental solution and fundamental velocities have simple expressions. These are the $x_{3}$ axis and the plane $x_{3}=0$, i.e., $\theta=0, \pi$, and $\theta=\pi / 2$. In fact, we find for $\theta=0$ and $\pi$

$$
\left.\begin{array}{l}
\hat{\varphi}=\frac{-1}{4 \pi|x|} \delta(t), \hat{w}^{*}=-\frac{1}{4 \pi \rho_{0}} \frac{x_{3}}{4 \pi|x|^{3}} \sin t, \\
\hat{u}_{1}=\hat{u}_{2}=0, \hat{u}_{3}=\frac{1}{4 \pi \rho_{0}} \frac{x_{3}}{|x|^{3}}\{\delta(t)-\sin t\},
\end{array}\right\}
$$

and for $\theta=\pi / 2$

$$
\begin{aligned}
& \hat{\varphi}=\frac{-1}{4 \pi|x|}\left[\delta(t)+\int_{0}^{t} \frac{J_{1}(\tau)}{\tau} d \tau\right], \quad \hat{w}^{*}=0, \\
& \hat{u}_{\alpha}=\frac{1}{4 \pi \rho_{0}} \frac{x_{\alpha}}{|x|^{3}}\left[\delta(t)+\int_{0}^{t} \frac{J_{1}(\tau)}{\tau} d \tau\right], \quad \alpha=1,2 ; \quad \hat{u}_{3}=0 .
\end{aligned}
$$

Thus, directly above and below the disturbance the vertical velocity is excited into a permanent oscillation at the buoyancy frequency, while in the plane the velocities $\hat{u}_{\alpha}$ oscillate about and tend to the values $\frac{1}{4 \pi \rho_{0}} \frac{x_{\alpha}}{|x|^{3}}$. It is not really surprising that the fluid injected at $t=0$ should try to squeeze into the lamina $x_{3}=0$ from which it came. 
Some of the properties of the fundamental solution that have just been noted are reflected immediately in the properties of the solutions (2.9) and (2.10).

The decomposition of $\hat{\varphi}$ and $\hat{\mathbf{u}}$ has its direct counterpart. In fact, we see that in general we may write the solution of (2.1) and (2.3) as $\varphi=\varphi^{\circ}+\varphi^{*}, w^{*}$, and $\mathbf{u}=\mathbf{u}^{\circ}+\mathbf{u}^{*}$, where

$$
\left.\begin{array}{l}
\varphi^{\circ}(x, t)=\int_{R^{3}} \frac{-1}{4 \pi|x-y|}\left\{\mu(y, t)-\frac{\partial h}{\partial y_{3}}(y, t)\right\} d y, \\
\varphi^{*}(x, t)=\int_{0}^{t} \int_{R^{3}} \hat{\varphi}^{*}(x-y, t-\tau)\left\{\mu(y, \tau)-\frac{\partial h}{\partial y_{3}}(y, \tau)\right\} d y d \tau, \\
w^{*}(x, t)=\int_{0}^{t} \int_{R^{3}} \hat{w}^{*}(x-y, t-\tau)\left\{\mu(y, \tau)-\frac{\partial h}{\partial y_{3}}(y, \tau)\right\} d y d \tau+\frac{h(x, t)}{\rho_{0}},
\end{array}\right\}
$$

and

$$
\left.\begin{array}{l}
\mathbf{u}^{\circ}(x, t)=\int_{R^{3}} \frac{1}{4 \pi \rho_{0}} \frac{x-y}{|x-y|^{3}}\left\{\mu(y, t)-\frac{\partial h}{\partial y_{3}}(y, t)\right\} d y, \\
\mathbf{u}^{*}(x, t)=\int_{0}^{t} \int_{R_{3}} \hat{\mathbf{u}}^{*}(x-y, t-\tau)\left\{\mu(y, \tau)-\frac{\partial h}{\partial y_{3}}(y, \tau)\right\} d y d \tau+\frac{h(x, t) \mathbf{k}}{\rho_{0}} .
\end{array}\right\}
$$

Clearly, the effect of a source distribution $\mu$ is two-fold. First there is an ideal fluid response given by the expressions for $\varphi^{\circ}$ and $\mathbf{u}^{\circ}$; then there is a slower long-lasting response due to buoyancy given by the expressions for $\varphi^{*}, w^{*}$, and $\mathbf{u}^{*}$. The latter depends, as the integrals show, on the history of $\mu$.

Suppose that $\mu$ is a source distribution that vanishes outside a fixed bounded region $G$ of space and has a total strength $M(t) \equiv \int_{G} \mu(x, t) d x$. By a standard line of argument ${ }^{5}$ it is clear that at distances from $G$ that are large compared to the diameter of $G$ the source distribution has very nearly the same effect as a concentrated source of strength $M$ located at the center of $G$. This leads naturally to an interest in a variety of concentrated source distributions, but discussion of this topic would lead too far afield; we shall pursue it elsewhere [4].

It is interesting that at large distances directly above and below $G$ one is led by (3.2) to expect very small horizontal velocities and vertical velocities close to

$$
u_{3}=\frac{1}{4 \pi \rho_{0}} \frac{x_{3}-y_{3}}{|x-y|^{3}}\left[M(t)-\int_{0}^{t} M(\tau) \sin (t-\tau) d \tau\right]
$$

where $y$ is the center of $G$. The second term in the brace is exactly the response of a simple oscillator having the buoyancy frequency and driven by a force $M(t)$.

The influence of the initial conditions through the source-like term $-\frac{\partial h}{\partial y_{3}}$ in (3.4) and (3.5) has the same decomposition into an instantaneous part and a slowly responding part. The terms in $\varphi^{\circ}$ and $\mathbf{u}^{\circ}$ as well as $h(x, t) / \rho_{0}$ in $w^{*}$ and $\mathbf{u}^{*}$ ring at the buoyancy frequency, and the latter with no change in shape from that given by the initial functions $f$ and $g$.

If $f$ and $g$ are smooth and vanish outside a bounded region $G^{*},-\partial h / \partial y_{3}$ has zero total source strength. Since $h$ vanishes outside $G^{*}$, the effect of such an initial disturbance dies off at large distances faster than that of a source distribution of nonzero total strength.

It is worthwhile to note that if $f$ and $g$ are independent of the vertical coordinate, then $\partial h / \partial y_{3}$ vanishes, so the total influence of the initial conditions is given by the term $h / \rho_{0}$ in $w^{*}$ and $u_{3}^{*}$. This 
can be seen directly from (2.1) and (2.2) as well. In fact, it is clear that the second of (2.1) is satisfied with $\mu \equiv 0$ by $\varphi \equiv 0$ and $w^{*}$ independent of $x_{3}$. Then the first of (2.1) and (2.2) imply

$$
w^{*}=f(x) \cos t+g(x) \sin t=h(x, t) / \rho_{0} .
$$

\section{The Fundamental Displacements}

This section is devoted to a brief examination of the displacements caused by the fundamental velocity field. The disturbance is taken to be at the origin, and for convenience it is scaled by the factor $\alpha$ so that the velocities are given by those of (2.4) multiplied by $\alpha$.

Since the velocity field is radial, each fluid particle is displaced radially by the flow depending on its original radius $r_{0}$ before the disturbance, its polar angle $\theta$, and time. In fact, the radial position $r$ of a particle satisfies the ordinary differential equation $d r / d t=\hat{u}_{r}$, which takes the form

$$
\frac{d r}{d t}=\frac{\alpha}{4 \pi \rho_{0}} \cdot \frac{1}{r^{2}} \hat{F}(\cos \theta, t)
$$

where $\hat{F}$ is given by

$$
\hat{F}(\cos \theta, t)=\delta(t)+\frac{\partial^{2}}{\partial t^{2}} t J_{0}(t \cos \theta)+\left[\frac{\partial}{\partial t} t J_{0}(t \cos \theta)\right] *\left[\frac{J_{1}(t)}{t}\right] x,
$$

The equation (4.1) is easily integrated to obtain

$$
r\left(r_{0}, \theta ; t\right)=\left[r_{0}^{3}+\frac{3 \alpha}{4 \pi \rho_{0}} F(\cos \theta, t)\right]^{1 / 3}
$$

in which the quantity $F$, defined by $F(\cos \theta, t) \equiv \int_{0}^{t} \hat{F}(\cos \theta, \tau) d \tau$, is given explicitly by

$$
F(\cos \theta, t)=\frac{\partial}{\partial t} t J_{0}(t \cos \theta)+\left[t J_{0}(t \cos \theta)\right] *\left[\frac{J_{1}(t)}{t}\right]
$$

From (4.4) simple expressions can be obtained for $F$ on the $x_{3}$ axis and in the plane $x_{3}=0$. These are

$$
F(\cos \theta, t)=\left\{\begin{array}{l}
\cos t, \theta=20, \pi, \\
J_{0}(t)+t \int_{0}^{t} \frac{J_{1}(\tau)}{\tau} d \tau, 2 \theta=\pi / 2
\end{array}\right.
$$

For ease in interpretation we take $\alpha=\frac{4 \pi}{3} \rho_{0}$ so that the coefficient multiplying $F$ in (4.3) is one. This is equivalent to an impulsive injection at $t=0$ of just enough fluid to fill a sphere of unit radius. Since $F\left(\cos \theta, 0^{+}\right)=1$, we have $r\left(0^{+}\right)=\left(r_{0}^{3}+1\right)^{1 / 3}$, so the first thing to happen is that the fluid pops out about the origin to accommodate the injected fluid. Subsequently, because $F(\cos$ $\theta, t)=1+0\left(t^{2}\right)$ for small positive $t$, the initial displacements begin to change very slowly.

We noted earlier that the velocity $\hat{\mathbf{u}}$ is singular at $x=0$ but does not have a net in- or outflow there after $t=0$. As a result, fluid is taken into the origin from some directions and given out in others. This is evidenced in the consequence of (4.5) that on the $x_{3}$ axis all the points that satisfy $r_{0} \leqslant 1$ before the flow starts are drawn into the origin during the interval $\pi / 2 \leqslant t \leqslant \pi$. Further 
out along the $x_{3}$ axis displacements oscillate up and down without ever reaching the origin. It also follows from (4.5) that the displacements in the plane $x_{3}=0$ grow without bound.

Vertical displacements in a stratified fluid are of considerable interest, for they are often the easiest to measure. We note that they can be obtained equally well by interpreting (4.3). If we designate a particle in terms of its original height $z_{0}=r_{0}-\cos \theta$ and $\theta$, the relation (4.3) also gives $z=r \cos \theta$ by

$$
z\left(z_{0}, \theta ; t\right)=\left[z_{0}^{3}+\frac{3 \alpha}{4 \pi \rho_{0}} \cos ^{3} \theta 2 F(\cos \theta, t)\right]^{1 / 3}
$$

In a subsequent article [4] we shall give a more detailed analysis of the displacement and velocity fields of the fundamental and other singular solutions.

\section{References}

[1] Courant, R., and Hilbert, D., Methods of Mathematical Physics, Vol. II (Interscience Publishers, 1962).

[2] Fox, D. W., Transient solutions for stratified fluid flow, notices, Amer. Math. Soc. 22, No. 5 (1975).

[3] Fox, D. W., An Initial Value Problem for a System of Equations for a Stratified Fluid (to appear).

[4] Fox, D. W., and Sigillito, V. G., Singular Solutions in Buoyant Flows (to appear).

[5] Miranda, C., Partial Differential Equations of Elliptic Type, Second Edition (Springer-Verlag, 1970).

[6] Sternberg, W. J., and Smith, T. L., The Theory of Potential and Spherical Harmonics (University of Toronto Press, 1946).

[7] Stoker, J. J., Water Waves (Interscience Publishers, 1957).

[8] Turner, S. J., Buoyancy Effects in Fluids (Cambridge University Press, 1973).

[9] Watson, G. N., Theory of Bessel Functions, Second Edition (Cambridge University Press, 1958).

(Paper 80B1-434) 